\title{
Testing of the mirrors for the Constellation-X spectroscopy $x$-ray telescope with a refractive null
}

\author{
J. P. Lehan ${ }^{\mathrm{a}, \mathrm{b}}$, T. Hadjimichael ${ }^{\mathrm{b}, \mathrm{c}}$, and C. Skocik ${ }^{\mathrm{b}, \mathrm{d}}$ \\ ${ }^{a}$ Center for Research and Exploration in Space Science and Technology, NASA Goddard \\ Space Flight Center, and Department of Physics, University of Maryland, Baltimore \\ County, 1000 Hilltop Circle, Baltimore, Maryland 21250 \\ ${ }^{b}$ NASA Goddard Space Flight Center, Greenbelt, Maryland 20771 \\ ${ }^{c}$ Ball Aerospace, 1616 McCormick Drive, Upper Marlboro, MD 20774 \\ ${ }^{d}$ ManTech Corporation, 12015 Lee Jackson Highway, Fairfax, Virginia 22033
}

\begin{abstract}
We present an introduction to the use of a refractive null lens for testing grazing incidence $\mathrm{x}$-ray mirrors for the Constellation-X mission. The singular role of mirror mounting in glass shell mirror metrology is also touched upon. We compare results achieved to date with mission requirements along with some of the unique properties of the null lens. Additionally, uses beyond mirror metrology are briefly discussed.
\end{abstract}

Keywords: Optical metrology, x-ray optics, Constellation-X, cylindrical optics, kinematic mounting

\subsection{INTRODUCTION}

The Constellation-X mission ${ }^{1}$ is a spectroscopic $\mathrm{X}$-ray mission to be flown in 2016 as a compliment to the current Chandra $\mathrm{x}$-ray observatory. The mission concept consists of a single spacecraft with four $1.3 \mathrm{~m}$, $\sim 15$ arcsec HPD soft X-ray telescopes (SXT) with $\sim 200$ nested, azimuthally segmented shells of grazing incidence mirrors.

Metrology of such x-ray telescope mirrors has been arduous. Although they are hyperboloids and paraboloids, the usual methods for testing these conic sections prove impractical because the annular sections are so far off axis. Instead, large numbers of line profiles parallel and perpendicular to the telescope axis have been measured and stitched together. Such an approach becomes unreliable for thin shell (foil or membrane) mirrors where vibration and self-weight distortion become an appreciable fraction of the metrology uncertainty. Under such circumstances, only a full surface metrology will allow one to deconvolve the various terms and determine the shape of the mirror in question. It is with this problem in mind that we designed a cylindrical null lens, discussed previously. ${ }^{2}$ We have since designed and built a lens housing and aligned the system ${ }^{3}$ and the system is now in regular use. The system is shown as used in Figure 1. We will briefly review some of the salient features of the design germane to the present discussion and then discuss results obtained to date.

\subsection{Refractive null lens and mirror metrology requirements}

The null lens provides certain information necessary for the quality assurance for the segmented x-ray telescope. These data must be capable of being measured to a fraction of the budgeted error for the mirror segment prescriptions. The quantities obtainable by the null lens are shown in Table I. 


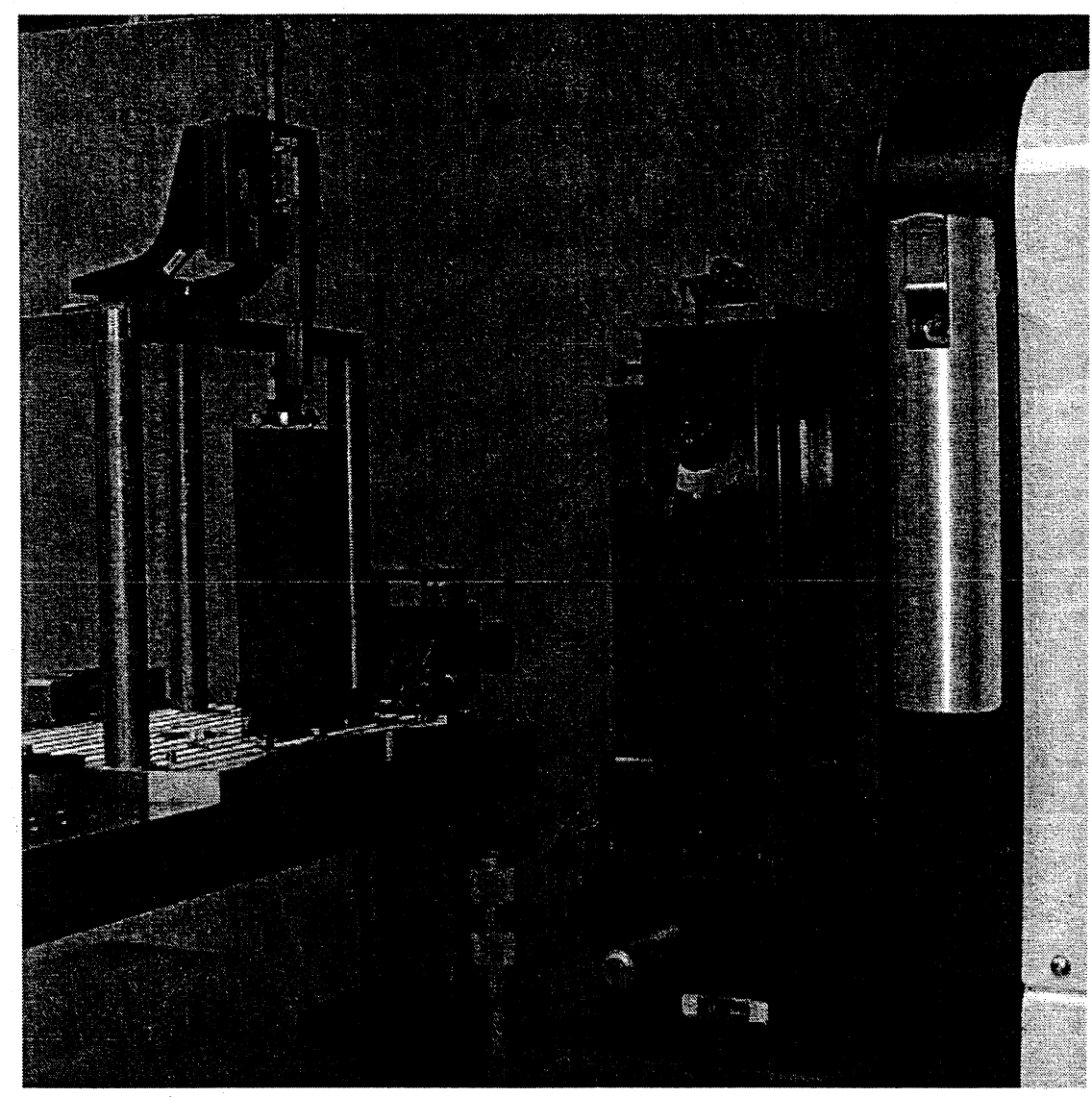

Figure 1: Null lens measuring mirror segment. Note Cantor tree mirror mount at left (see text), cylindrical null lens at the right-center and strobe Fizeau at far right foreground.

In addition to the quantities listed in Table I, the null lens is of great utility in measuring the gravity distortions. Terrestrial metrology on the 500:1 aspect ratio mirrors will inevitably be influenced by gravity distortions. Only a full surface metrology technique unambiguously measures the surface deformations allowing the testing of mounts to minimize the gravity distortion.

Table I: Terms from the Constellation-X error budget ${ }^{4,5}$ derivable from the null lens measurements and determined uncertainties as of this writing.

\begin{tabular}{|l|c|c|c}
\hline \multicolumn{1}{|c|}{ Term } & Error Allocation & $\begin{array}{c}\text { Metrology } \\
\text { Uncertainty }\end{array}$ & Notes \\
\hline Roundness & $5 \mu \mathrm{m} \mathrm{RMS}$ & $0.06 \mu \mathrm{m}$ RMS & 1 \\
\hline$\Delta \Delta \mathrm{R}$ & $0.5 \mu \mathrm{m} \mathrm{RMS}$ & $0.04 \mu \mathrm{m} \mathrm{RMS}$ & 2 \\
\hline Average Axial Sag & $10 \mu \mathrm{m} \mathrm{P}-\mathrm{V}$ & $0.004 \mu \mathrm{m} \mathrm{P}-\mathrm{V}$ & 2 \\
\hline Axial Sag Deviation & $0.1 \mu \mathrm{m} \mathrm{P}-\mathrm{V}$ & $0.08 \mu \mathrm{m} \mathrm{P}-\mathrm{V}$ & 2 \\
\hline Axial Slope irregularity & $2.4 \operatorname{arcsec} \mathrm{RMS}$ & $0.05 \operatorname{arcsec} \mathrm{RMS}$ & 2 \\
Circumferential slope error & $\begin{array}{c}1.6 \operatorname{arcsec} \mathrm{RMS} \\
\text { (shell dependent) }\end{array}$ & $0.06 \operatorname{arcsec} \mathrm{RMS}$ & 2 \\
\hline
\end{tabular}

Notes:

1. Estimated from optical model

2. Measured 


\subsection{Characteristics of the cylindrical refractive null lens}

We have discussed the design of the lens in a previous publication and the interested reader is referred to Ref. 2 for the details. We note that a single refractive cylindrical null lens will measure all the mirrors in the telescope despite the plethora of cone angles involved. This makes the refractive solution more than an order of magnitude less expensive than alternative approaches such as diffractive nulls. This advantage is also a drawback since the cone angle is not then determined uniquely. This disadvantage can be overcome (discussed at the end of the paper). An additional advantage making the refractive null solution attractive is the time savings realized in calibrating a single null corrector rather than hundreds.

Since our previous report on the design, the null lens has been fabricated; a housing designed and built; lens mounted and aligned; ${ }^{3}$ and it is now in regular use. The present lens has a field of view of only 36 degrees, but a second lens is being fabricated with a field a view greater than $60^{\circ}$. This larger field of view will allow either inner or outer mirror segments to be measured in a single exposure.

The lens provides quality assurance and feedback for the glass forming (slumping) process. ${ }^{6.7}$ It is intended to cover the spatial period region from $200 \mathrm{~mm}$ to about $1 \mathrm{~mm}$.

The null lens exhibits an anamorphic magnification of the part under test. Parallel to the lens axis, the magnification is unity whereas perpendicular to it, the magnification is a function of the radius of the part under test. For the data presented here the ratio of azimuthal to axial magnification is very close to $1 / 3$. The anamorphic magnification also results in the sampling of the mirror being anamorphic as well. Since the radius is not determined by the measurement itself, it is most accurate to express the surface maps axially in terms of $\mathrm{mm}$ but azimuthally in terms of angle. We have not corrected the surface maps in this paper for this anamorphic magnification.

\subsection{The role of mounting for glass shell mirrors}

The self-weight distortions and vibrations complicate the mirror shape determination. The low stiffness of the glass membrane mirrors results in large amplitude, low-frequency vibrations $(<50 \mathrm{~Hz})$. This was discussed in detail in a previous publication. ${ }^{8}$ Given the self-weight distortion and vibrations, great care must be taken in the design of the mount used for metrology. First, it must support the mirror to minimize gravity distortions. Second, it needs to suppress (or control the shape of) the vibrations. Finally, it must allow for precision manipulation of the mirror to align to the gravity field and null lens. And it should accomplish these without over constraining the mirror. The properties of an ideal mount are summarized in Table II after Ref 8.

\begin{tabular}{|c|}
\hline Table II: Characteristics of an ideal metrology fixture \\
\hline Low self-weight gravity distortion \\
\hline No twist of the part under test \\
\hline${ }^{1}$ \\
\hline Part invertible \\
\hline Repeatable positioning \\
\hline Minimal obscuration of aperture \\
\hline Minimal obstruction of access to surface under test \\
\hline Provides fiducials \\
\hline Minimizes vibration $^{2}$ \\
\hline
\end{tabular}

1. More generally doesn't over constrain the mirror under test

2. More generally minimizes effects of vibration on the measurement

To solve the Herculean task of the mount design we took our inspiration from kinematic design. Accounting for the degrees of freedom of the mirror, we ended up with a system somewhat akin to a twodimensional Whiffle tree, which we have nicknamed a Cantor tree (given its resemblance to the Cantor set when sketched - see Figure 2). It consists of bearings and fulcrums along the bottom and bearings only along the top. The bearings accommodate the possibility of locally varying slopes along the perimeter of 


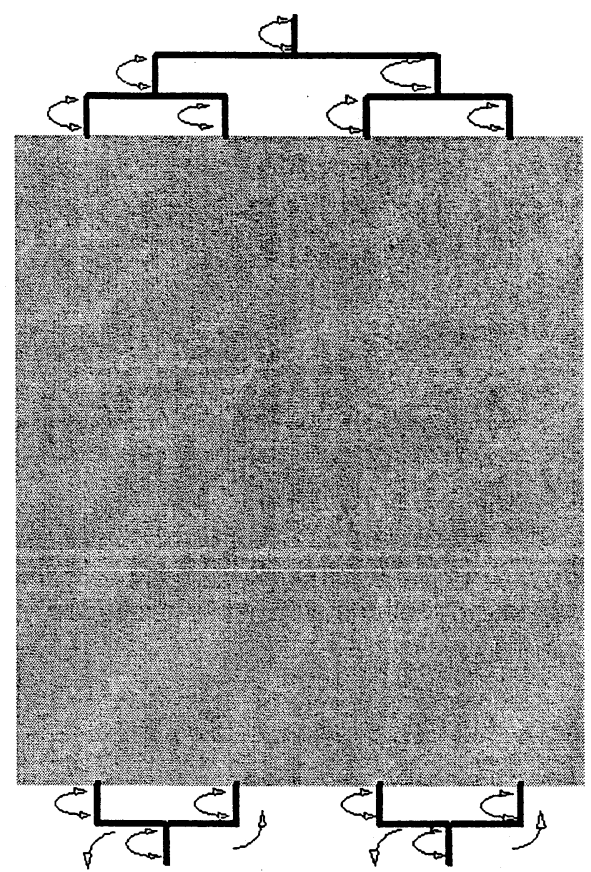

$\leftarrow$ Figure 2: Schematic of Cantor tree kinematic mount holding a mirror. Positions marked with bearings that rotate in and out of the plane of the paper. The bottom two branches also rock in the plane of the paper as indicated. The $\mathbf{g}$ indicates the assumed gravity vector for the design. Note that the part can be inverted and remounted. Top contact points are guided whereas one contact point is fixed on the bottom while the rest are guided.

the mirror without distorting the mirror. One of the bottom points of contact is fixed and all the top points are guided. It is shown schematically in Figure 2 for four points of attachment at the top and bottom. (A realization with two points of attachment on the top and bottom is seen in the Fig. 1.) Note that it can be reduced to a three-point kinematic mount for precise manipulation of the mirror.

The resemblance to a Whiffle tree ceases when the part-mount combination dynamic behavior is considered. The contact points and branches are optimized to tune the frequency of vibration and the mode shapes of the mirror while simultaneously minimizing the gravity distortion.

For the purpose of metrology, the mode shape is chosen so that the vibration will have a minimum impact on the metrology results. (We have found that we cannot completely suppress the mirror vibrations without over constraining the mirror and thus distorting it; so the mirror is allowed to vibrate during measurement.) For the mirror segments we are measuring, this means the ideal modes are as close to purely azimuthal as possible.

We note that the general Cantor tree concept can be optimized differently than we have chosen to do here. For instance, one could simultaneously minimize of both the static and dynamic deflections.

In addition to vibrational mode shaping, we also employ the usual precautions to minimize the vibrational amplitudes resulting from environmental influences by using a high quality air table, etc. We have found, however, that this approach is insufficient (with any reasonable effort) for vibrations. With our thin mirrors the mirror vibrational amplitudes are unacceptably large compared with our desired metrology precision even for the small vibrational source amplitudes that transmit through the air table.

\subsection{RESULTS AND DISCUSSION}

Given the variable nature of the glass shell mirrors as discussed above, we average over the mirror vibrations by employed a $250 \mathrm{~mm}$ aperture 4D Technologies FizCam®1500 to strobe the part interferometrically thereby elucidating the static (unvibrating) shape. A large number (typically $>100$ ) of individual interferograms are taken at successive times $(67 \mathrm{msec}$ between frames with a $0.2 \mathrm{msec}$ exposure). The frames are averaged to yield the vibration-free shape. Figure 3 shows the shape of the first mode of vibration for the mirror in the mount captured by the refractive null-FizCam combination.

From the resultant unvibrating (average) shapes, obtained in carefully chosen orientations relative to gravity, and with comparison to FEM models, we can extract the shapes free of gravity distortions. Thus, the as-formed shape can be determined for feedback to the forming process. This work is in its early stages as of this writing. 


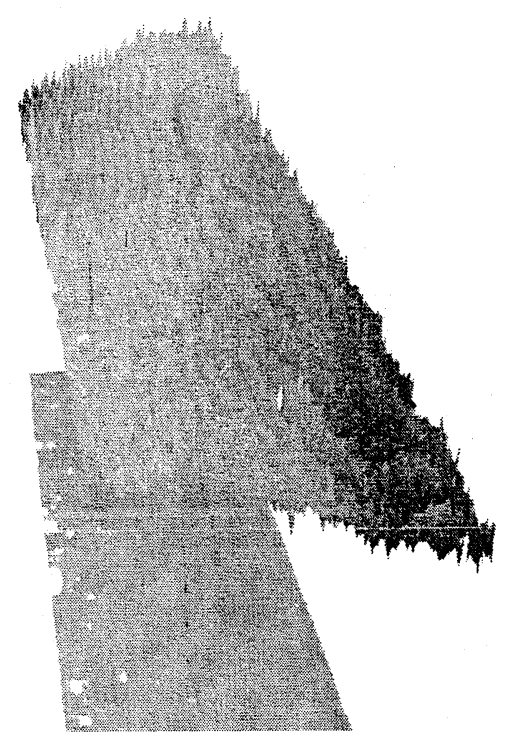

$\leftarrow$ Figure 3: This figure illustrates the shape of the first vibrational mode of the part in the Cantor tree mount. (This figure was generated by taking a single surface map and subtracting the average of all 100 to bring out the mode shape.) The P-V is 60 $\mathrm{nm}$.

Other possible uses of the null-lens-strobe interferometer combination outside the standard metrology for the Constellation-X mission are numerous. For instance, the null lens can be employed to quantify the distortions introduced by the telescope housing to optimize the opto-mechanical design. It can also be employed to study the vibrational characteristics of the mirrors prior to pre-launch vibration and acoustic testing.

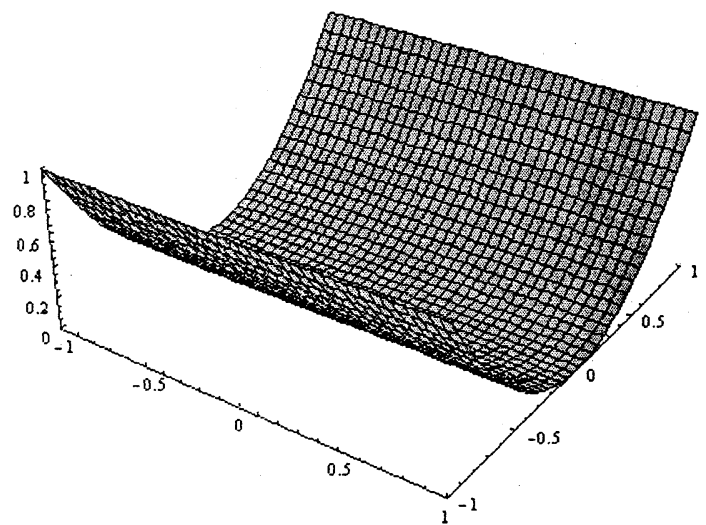

(a)

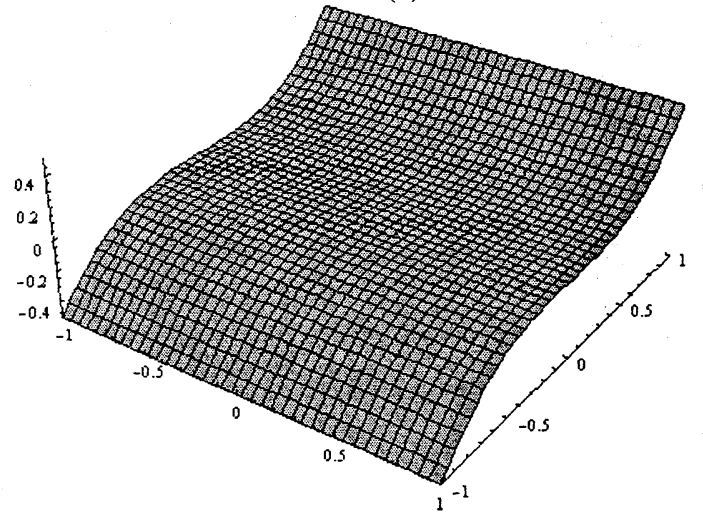

(c)

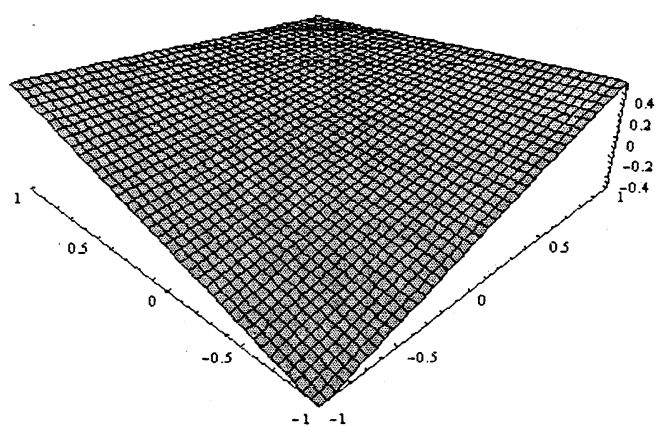

(b)

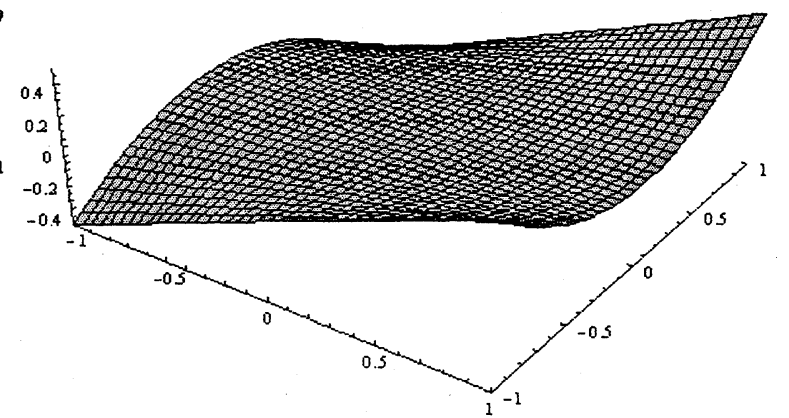

(d)

Figure 4: Misalignment aberrations for a cylindrical (or conical) system [normalized]. (a) defocus, (b) twist (clocking of lens and part axes) (c) coma ( $1 \mathrm{D}$ coma from displacement of axes) (d) conical (tip of axes yielding defocus varying as a function of axial position).

The cylindrical null lens measurements, however, are not absolute. Figure 4(a)-(d) illustrate the aberrations resultant from misalignments between the mirror under test and the lens. As any of these shapes are also conceivable in a mirror as fabricated, measurements in different lens-part orientations should be made to 
determine the source of the observed error (mirror or misalignment). A possibly better method of assuring that such a shape is in the mirror is to move the lens a known amount between two successive measurements, which introduces a known amount of aberration. Several such moves allow one to determine if the shape is in the part or merely a misalignment. One part error that cannot be determined by this method is shown in Figure 4(d) and is called conical aberration. This is an error in the cone angle of the part (assuming the lens is oriented exactly at the proper cone angle). This error in mirror fabrication can always be accommodated (or at least its first-order component) by tilting the lens relative to the interferometer. Although this characteristic of the null lens allows any cone angle to be measured, it does not tell you what that cone angle is (see the extension discussion below for a way out of this conundrum).

Figure 5 shows a typical result for the average figure measurement. This represents the vibration-free figure after the removal of the tilted cylindrical wavefront (conical wavefront). We averaged 225 individual measurements to obtain this result. The large number assures a good sampling of the random (in amplitude, time and frequency content) environmental sources of vibration perturbing any individual realization of the part shape in time.

Figure 6 shows the difference between two such measurements (with 75 individual surface maps making up each average) indicating the excellent repeatability obtained by the averaging process. Most of the error is due to residual air movement during the time between measurements (about 2 minutes here).

\section{SUMMARY AND CONCLUSIONS}

A refractive null corrector has been designed and fabricated that allows large area metrology for grazing incidence $x$-ray mirrors. This enables one to examine the dynamics of the membrane mirrors, which, in turn, allows one to design and test mounting structures with the small amplitude displacements critical for surviving launch. Additionally, the static shapes can be determined from which the on-orbit shapes may be derived.

The precision of the system is currently limited by mirror fixturing. Since the fabrication technology for cylindrical optics of this size is mature, the system is scalable in both azimuthal and axial dimensions so much larger mirror segments could be measured by this method.

In conclusion, the addition of a refractive null corrector is a powerful weapon in the arsenal of metrology needed for the Constellation-X mission.

\section{EXTENSION OF THE NULL LENS TO THE MEASUREMENT OF OTHER ERROR BUDGET TERMS}

\subsection{Cone angle measurement}

The null lens can accommodate a wide range of cone angles. Although this allows any cone angle in the telescope to be accommodated, it does not tell you what that cone angle is. There is a way out of this corner. This is merely the addition of an angular fiducial to the lens housing. A convenient angular fiducial with the requisite fidelity is a variable line space grating or CGH. This can be a small strip glued to the housing.

Then one only need determine the zero of the angular scale. This is readily done by obtaining a cat-eye interferogram from a very good flat and noting the position of the CGH return in the field of view.

\subsection{Average radius measurement}

The average radius of the part can be measured akin to radius measurements with a radius slide for spherical optics. It behooves one to start the measurement with the normal full surface null measurement. 

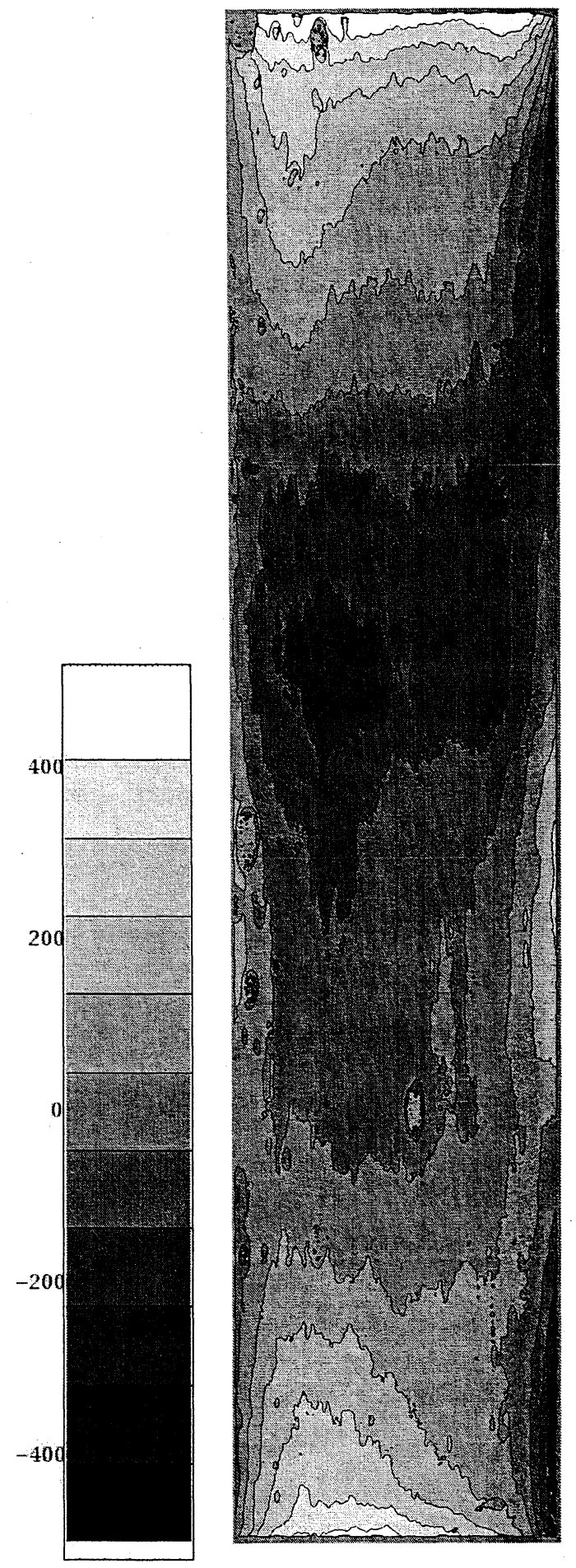

Figure 5: Typical data obtained from the cylindrical null lens and strobe Fizeau interferometer combination. Note that the axial sag of the part is intentional. Drop outs in the data are local high slope regions left from the forming process, which would primarily contribute, to a loss of effective area in the telescope.

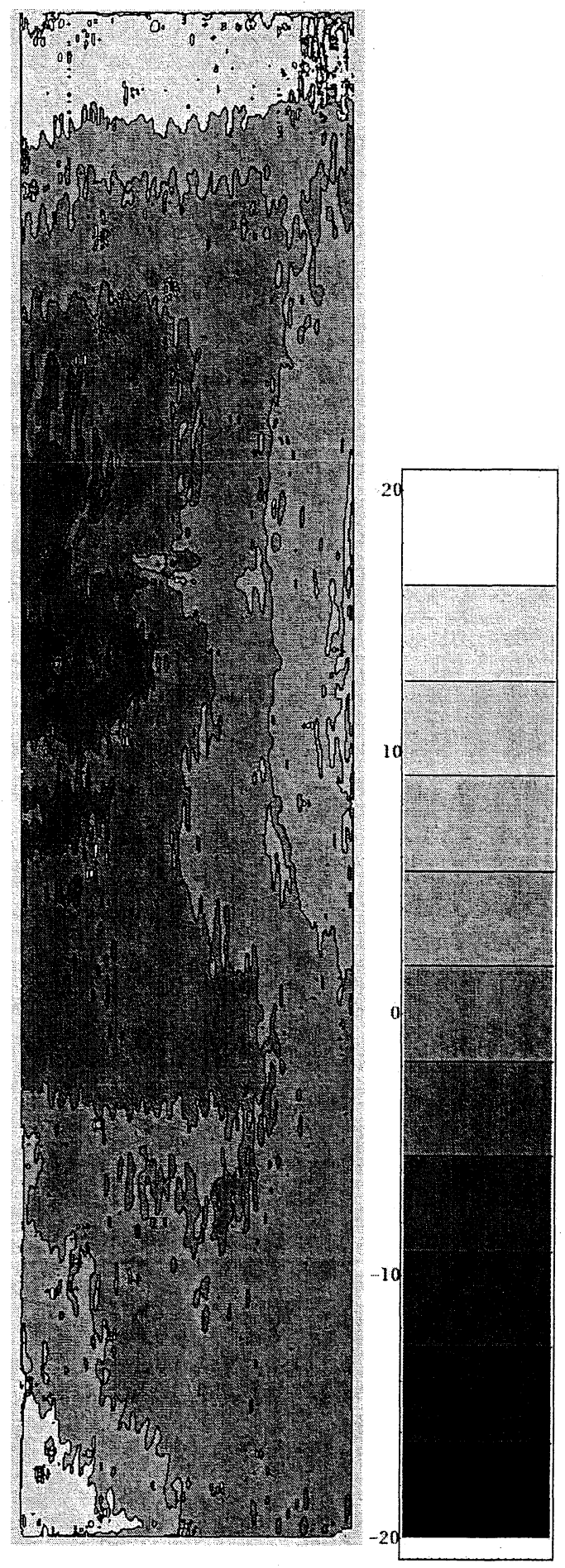

Figure 6: Difference plot between two subsequent averages for $100 \%$ of the null lens clear aperture. RMS error is $5.9 \mathrm{~nm}$. Note the dominant residual seen is probably due to air currents. 
This assures that the lens is centered on the part. Then the null lens is slid forward on the slide until it is focused on the centerline of the part in a cat's-eye interferogram. The distance the lens travels along the slide is the radius of the part.

The measurement is very sensitive to side-to-side travel (as in a standard spherical optics radius measurement). Unlike in a standard radius measurement though, the up-and-down motion of the stage is not as critical. (The sensitivity is proportional to the half-cone angle of the part under test.) Clocking of the lens (twist of the rail) is a source of error too. It can be tracked by examining the axial sag, which should be identical at the centerline in both the null and cat's-eye measurements.

\section{ACKNOWLEDGEMENTS}

This work is funded through the Constellation-X technology development program. In particular, we wish to thank Optimax Systems for fabrication of the lens, especially Mr. Joe Serio for the interferograms of the surfaces as fabricated; and Mr. Rick Plympton and Mr. Alan Gould for their general support and useful insights. We also wish to thank Mr. Dale Arbogast for his outstanding prototyping of the Cantor Tree mount; Mr. Thomas Van de Castle for his hard work taking the data; Dr. Dave Content for his unflagging support and useful discussion; and Dr. William Zhang for useful discussions. The mention of trade names or commercial products does not constitute endorsement by the authors or NASA.

\section{REFERENCES}

1 For an overview of the Constellation-X mission online see http://constellation.gsfc.nasa.gov

2 J. P. Lehan, T. Hadjimichael, D. A. Content, and W. W. Zhang ;"Design and fabrication of refractive nulls for testing the segmented mirrors of the Constellation-X spectroscopy x-ray telescope (SXT)," Proc. SPIE 5900, 59001D (2005).

3 J. P. Lehan, T. Hadjimichael, and D. A. Content, "Simple method of aligning cylindrical air-spaced elements," Opt. Eng. 46, 070503 (2007).

${ }^{4}$ W. A. Podgorski, J. Bookbinder, D. A. Content, W. N. Davis, M. D. Freeman, J. H. Hair, S. M. Owens, R. Petre, P. Reid, T. T. Saha, J. W. Stewart, and W. W. Zhang, "Constellation-X spectroscopy X-ray telescope optical assembly pathfinder image error budget and performance prediction," Proc. SPIE 5168, 318 (2004).

${ }^{5}$ P. Reid, R. Cameron, L. Cohen, M. Elvis, P. Gorenstein, D. Jerius, R. Petre, W. Podgorski, D. Schwartz, and W. Zhang, "Constellation-X to Generation-X: evolution of large collecting area moderate resolution grazing incidence $\mathrm{x}$-ray telescopes to larger area, high resolution, adjustable optics," Proc.SPIE 5488, 325 (2004).

${ }^{6}$ W. W. Zhang, K.-W. Chan, D. A. Content, J. P. Lehan, R. Petre, T. T. Saha, M. Gubarev, W. D. Jones, and S. L. O'Dell, "Development of lightweight $\mathrm{x}$-ray mirrors for the Constellation-X mission," Proc. SPIE 6266, 62661V (2006).

7 W. W. Zhang, et al, "Constellation-X mirror technology development status and plan,", Proc. SPIE 6688 6688-01 (2007).[These Proceedings]

${ }^{8}$ D. A. Content, D. Colella, T. Hadjimichael, J. P. Lehan, J. McMann, P. B. Reid, T. T. Saha, and W. W. Zhang, "Optical metrology for the segmented optics on the Constellation-X spectroscopy X-ray telescope," Proc. SPIE 5488, 272 (2004). 